\title{
Corrigendum to the paper "HERMITIAN FORMS OVER DIVISION ALGEBRAS OVER REAL FUNCTION FIELDS"
}

\section{Nguyen Quoc Thang}

We make some corrections to the paper "Hermitian forms over division algebras...", Bd. 78 (1993), pp. 9 - 35.

In the previous paper [T] we gave a classification of hermitian forms over the real function field $k=\mathbf{R}(t)$ and its completions $k_{v}$ with respect to valuations $v$ trivial on $\mathbf{R}$. Unfortunately in the local case the arguments given for cases $\mathbf{A}$ and $\mathrm{D}$, in general, were not correct. Therefore the resulting local and local-global classifications obtained were incorrect. I would like also to thank Dr. D. Hoffmann for pointing out these mistakes and the referee for useful comments. Here we would like to make necessary corrections to [T]. We keep the same notation used there, except that in the first paragraph, $J$ is not the standard involution of a quaternion division algebra $D$ (with basis $\{1, i, j, i j\}$ ). All hermitian forms will be hermitian forms with respect to $J$, with values in $D$.

I. Local classiflcation of forms of type D. Assume that $D$ is of center $k_{v}, i^{J}=i, j^{J}=-j$. It is well-known (see [S2]) that, by scaling, forms of type D over $D$ can be considered as hermitian forms with respect to $J$. By $O_{D}$ (resp. $\mathrm{p}_{D}$ ) we denote the valuation ring of $D$ (resp. the maximal ideal of $\left.O_{D}\right) ; O_{D}=\{d \in D: v(N r d(d)) \geq 0\}$, $\mathrm{p}_{D}=\{d \in D: v(N r d(d))>0\}$. Let $\bar{D}=O_{D} / \mathrm{p}_{D}$ and $\pi$ (resp. $\left.t\right)$ be an uniformizing element of $k_{v}$ (resp. of $D, t \in \mathrm{p}_{D}$ ). We may assume that $v$ is real. Then parts $a$ ), $b$ ), c) of Theorem 4.1.1 of [T] are true only in the case $D=(-1,-1)$ and incorect in other cases, while part $d$ ) remains true. The reason is that we have used a result of $[P]$, which is true only in the case $D=(-1,-1)$. Therefore the resulting Corollary 4.1.2 and Theorem 4.1.5 are true only in this case. Using the results of [S1], it can be shown that Theorems 4.1.1 and 4.1.5 should be replaced by the following. 
Theorem 1. 1) Let $D=(-1,-1)$. Then $W^{1}(D, J) \simeq \mathbf{Z} / \mathbf{2 Z} \times \mathbf{Z} / \mathbf{2 Z}$. Any anisotropic $J$-hermitian form is equivalent to either $(i),(\pi i)$ or $(i, \pi i)$. A two-dimensional $J$-hermitian form $F$ is isotropic if and only if $F \simeq(z, \lambda z)$, where $\lambda \equiv \pm 1\left(\bmod . k_{v}^{* 2}\right)$ and $z$ is a J-symmetric element of $D$. Any J-hermitian form of rank 3 represent any skew-quaternion of $D$.

2) Let $D=(\pi,-1)$. Then $W^{1}(D, J) \simeq \mathbf{Z} \times \mathbf{Z} / \mathbf{2 Z}$. Any anisotropic $J$ hermitian form over $D$ is equivalent to a form $(\epsilon, \ldots, \epsilon) \perp p t(1)$, where $\epsilon= \pm 1$ and $p=0$ or 1 .

Proposition 4.1.4 of [T] remains correct and from above it follows that Corollary 4.1 .2 of [T] should be replaced by the following

Corolary. We have the following exact sequence of pointed sets

$$
1 \rightarrow \mu_{2} \rightarrow \mathrm{H}^{1}\left(k_{v}, \mathrm{SU}(F)\right) \rightarrow \mathrm{H}^{1}\left(k_{v}, \mathrm{U}(F)\right) \rightarrow E \rightarrow 1,
$$

where $E=1$ (resp. $\left.\mu_{2}\right)$ if $D \simeq(-1,-1)($ resp. $D \not(-1,-1))$.

II. Local classification of forms of type A. Let $D$ be a quaternion division algebra over $K=k_{v}(\sqrt{a})$ with a $K / k_{v}$-involution $J$, where $v$ is a real valuation. By Albert's Theorem [S2, Theorem 8.11.2, p. 314] we may assume that $D=(\alpha,-1) \otimes K$, where $\alpha \in k_{v}$. Thus $D$ is a division algebra iff either $\alpha \equiv-1\left(\bmod . k_{v}^{* 2}\right)$ or $\alpha \equiv-\pi\left(\bmod . k_{v}^{* 2}\right)$ and $a \equiv \pi\left(\bmod . k_{v}^{* 2}\right)$, where $\pi$ is a fixed uniformizing element of $k_{v}$. Hence in all cases $D / K$ is unramified, $K / k_{v}$ is ramified and we may choose $t=\sqrt{\pi}$ to be an uniformizing element of $K$ and $D$ (see [T, p. 28]). Let $O_{v}$ (resp. $\mathrm{p}_{v}$ ) be the ring of $v$-integers of $K$ (resp. maximal ideal of $O_{v}$ ). We use also the notation $\bar{J}$ and $\bar{J}_{t}$ as in [S1]. Theorem 5.1.1 of [T] is incorrect because $W^{\mathbf{1}}(\bar{D}, \bar{J}) \simeq \mathbf{Z}$ (but not $\mathbf{Z} / \mathbf{2 Z}$ ), while $W^{\mathbf{1}}\left(\bar{D}_{,}, \bar{J}_{t}\right) \simeq \mathbf{Z} / \mathbf{2 Z}$. Thus those statements concerning forms in Theorem 5.1.3 are incorrect also. Using the results of [S1], one can show that Theorems 5.1.1 and 5.1.3 should be replaced by the following.

Theorem 2. Any anisotropic J-hermitian form over $D$ is equivalent to a form $(\epsilon, \ldots, \epsilon) \perp \delta$ ti, where $\epsilon \in\{ \pm 1\}, \delta \in\{0,1\}$ and $W^{1}(D, J) \simeq$ $\mathrm{Z} \times \mathbf{Z} / 2 \mathrm{Z}$. If $\alpha \equiv-1\left(\bmod . K^{* 2}\right)\left(\right.$ resp. $\left.\not \equiv-1\left(\bmod . K^{* 2}\right)\right)$ then $\operatorname{Nrd}\left(D^{*}\right)$ 
$=K^{* 2}$ and $N_{K / k_{v}}\left(N r d\left(D^{*}\right)\right)=k_{v}^{* 2}\left(\right.$ resp. $\left.\left[k_{v}: N_{K / k_{-}}\left(N r d\left(D^{*}\right)\right)\right]=2\right)$. Therefore there are only four (resp. two) possibilities for determinants of forms of type $\mathrm{A}$ over $k_{v}$.

\section{Local-global classification.}

Theorems 4.2.1, 4.2.2, and 4.2.3 and Proposition 4.2.6 in the LocalGlobal Classification of [T] as well as their proofs remain correct, but the corrections in I and II necessitate the following alterations.

1) The correct formulation of Theorem 4.2.4 is as follows.

Theorem 3. Let $F$ be a form of type $D$ of rank $n$ with values in a quaternion division algebra over $k=\mathbf{R}(t)$.

i) If $D=(-1,-1)$ and $n \geq 2$ then $F$ is isotropic.

ii) If $D \not(-1,-1)$ and $n \geq 3$, then the Strong Hasse Principle holds for $F$.

2) Regarding Proposition 4.2.7, one should add the condition " $D \simeq$ $(-1,-1)$ " at the beginning.

3) Regarding Theorem 5.2.1, the last two sentences of $b$ ) should be removed due to corrections in II.

4) Regarding Proposition 5.2.2, the last sentence should be removed. The proofs remain essentially the same.

\section{References}

[P] B. Pollak, The equation tat $=6$ in a quaternion algebra, Duke Math. J. 27, $261-271$ (1961)

[S1] W. Scharlau, Klassifikation hermitescher Formen über lokalen Körpern, Math. Ann., 186, $201-208$ (1970)

[S2] W. Scharlau, Quadratic forme and Hermitian forms, Berlin-Heidelberg-New York, Springer-Verlag, 1985

[T] N. Q. Thang, Hermitian forme over division algebras over real function fielde, Manuac. Math., 78, 9 - 35 (1993).

Nguyen Quoc Thang

Department of Pure Mathematic:

University of Waterloo, Waterloo

Ontario, Canada N2L 3G1

E-mail: nqtnguyen Qjeeves.uwaterloo.ce 